\title{
Internal consistency and correlation of the adverse childhood experiences, bully victimization, self-esteem, resilience, and social support scales in Nigerian children
}

\author{
Morenike Oluwatoyin Folayan ${ }^{1,2^{*}}$ (D) Olakunle Oginni ${ }^{1,2}$, Olaniyi Arowolo ${ }^{1}$ and Maha El Tantawi ${ }^{3}$
}

\begin{abstract}
Objectives: We evaluated the internal consistencies and the correlation between measures of adverse childhood experiences (ACE), bully victimization, self-esteem, resilience, and social support in children/adolescents in Nigeria.

Results: The Cronbach's alphas were 0.67 for the ACE Questionnaire; 0.79 for the victimization subscale of the Illinois Bully Scale; 0.60 for Rosenberg's self-esteem scale; 0.81 for Connor-Davidson resilience scale; and 0.93 for multidimensional perceived social support scale. Social support was negatively correlated with ACE $(r=-0.21)$ and bully victimization $(r=-0.16)$ and was associated with higher self-esteem $(r=-0.29)$ and higher resilience $(r=0.15)$. Likewise, higher resilience was associated with fewer ACE $(r=-0.07)$, higher self-esteem $(r=-0.21)$, and higher bully victimization $(r=0.13)$. Higher self-esteem was associated with fewer ACE $(r=0.25)$ and lower bully victimization $(r=0.16)$, whereas bully victimization was positively correlated with ACE $(r=0.20)$. The correlations were all statistically significant.
\end{abstract}

Keywords: Mental health, Internal consistency, Adverse childhood experiences, Bully victimization, Self-esteem, Resilience, Social support, Psychometric scales, Children, Adolescents, Nigeria

\section{Introduction}

Poor mental health is a major health problem affecting children and adolescents globally. Mental health disorders have great impact on children ages 5 to 14 years in North America and Europe, and they are a growing problem in other regions of the world due to the complex change in patterns of health and disease and the interaction with demographic, economic, and sociologic determinants [1].

Factors that affect children's and adolescents' mental health include adverse childhood experiences (ACE)

\footnotetext{
*Correspondence: toyinukpong@yahoo.co.uk

${ }^{2}$ Department of Child Dental Health, Obafemi Awolowo University, Ile-Ife, Nigeria

Full list of author information is available at the end of the article
}

and bully victimization. ACE include physical or sexual abuse; domestic violence; exposure to violence in the community; neglect; the absence or limited availability of a caregiver; poverty; and food insecurity [2]. These experiences affect emotional expression and increase the use of maladaptive emotion-regulation strategies [3, 4]. These coping measures can result in psychopathology such as depression, anxiety, aggressive behavior, post-traumatic stress disorder, and substance abuse [5-7]. Children and adolescents who experience bully victimization may face these mental health challenges also in adult life [8-11].

Factors that protect against mental health problems include self-esteem, resilience, and social support. Selfesteem is the ability to hold a favorable attitude towards one's self. High self-esteem is associated with fewer symptoms of anxiety and depression [12]. Resilience, 
which is the ability to positively adapt to adversity, is associated with good mental health [13-15]. Also, previous studies showed that the support that individuals perceive they get through social interactions moderates the impact of ACE and bully victimization through a buffering effect that reduces psychological distress when stressful situations develop [12, 16, 17].

Though the impact of ACE, bully victimization, selfesteem, resilience and social support on mental health is assumed to be a global phenomenon [18], the interactions between these variables may vary with culture (beliefs, norms and values) [19]. Most of the studies on ACE, bully victimization, self-esteem, resilience, social support, and mental health have been conducted in Western countries, which are generally individualistic societies and differ in many ways from the collectivist societies of Africa [20].

ACE, bully victimization, and adolescents' concern with self-esteem are also problems in Nigeria [21-24], and mental health difficulties are a growing problem among adolescents in the country [25]. It is important to conduct more studies, using validated tools, to elucidate the correlations between variables associated with mental health problems in settings and adolescent populations in Nigeria. The aim of this study was therefore to assess the internal consistency of tools used to measure ACE, bully victimization, self-esteem, resilience, and social support in a population of in-school children and adolescents in sub-urban Nigeria. We also determined how the constructs correlated with one another. We hypothesized that ACE and bully victimization would be associated negatively with social support, resilience, and self-esteem.

\section{Main texts}

Data were collected as part of a cross-sectional study that determined the association between caries and nutritional status in 6-16-year-old children attending private and public primary and secondary schools in Ife Central Local Government Area, Ile-Ife, Osun State, Nigeria. Data were collected from October to December 2019. Children at least 6-year old were recruited to ensure they had the cognitive ability to respond to the interviewer-administered questionnaire [26, 27]. Recruiting children 6-10-year-old for study participation further reduces the risk for recall bias, a major challenge when studying the impact of ACE and bullying in older individuals [28]. Children and adolescents with special health-care needs, those who were ill, and those who had fasted within 3 months before data collection, were excluded from the study.

\section{Sample size and sampling procedure}

The sample size for the primary study was 1209 . Post-hoc power analyses using the formula by Metcalfe [29] indicated a power of $80 \%$ given a population prevalence rate of $13.9 \%$ for caries in children [30]. The sample was collected through a multi-stage cluster sampling technique that recruited 6-10-year-old children from primary schools, and 11-16-year-old children from secondary schools. Twenty primary schools ( 3 public, 17 private) and 10 secondary schools (2 public, 8 private) were randomly selected from the list of schools in the local government based on the ratio of public/private primary and public/private secondary schools. Data were collected from pupils using tools written in English and administered by interviewers; English is the language of instruction in schools in Nigeria.

Adverse childhood experiences were measured with the 10-item ACE Questionnaire, which provides a measure of cumulative life stress experienced during childhood [31]. The response to each of the 10 questions is either 'yes' or 'no', with possible scores of 0 to 10 . The higher the score the more adverse experiences the child has faced. Although the instrument had not been validated for use in Nigeria, validation in other countries indicated that it had good psychometric properties, with high correlation between mental and physical health measures and childhood trauma inventories [32].

Childhood bully victimization was assessed with the victimization subscale of the Illinois Bully Scale [33]. The subscale consists of four questions that measure both physical and verbal victimization that individuals experience from or by peers. The responses to each question ranged from never (scored 0) to 1-2 times (1), 3-4 times (2), 5-6 times (3), and 7 or more times (4). The responses were summed to derive a total score that ranged from 0 to 16 . The verbal bullying, physical bullying, and bully victimization subscales of the Illinois Bully Scale were validated among 394 pupils in two secondary school in semi-urban southeast Nigeria. The Cronbach's alphas for the whole scale and the victimization subscale were 0.84 and 0.78 , respectively [17].

Self-esteem was assessed with the 10-item Rosenberg's self-esteem scale that evaluated whether respondents' perceptions of themselves were positive or negative. Items were scored on a Likert-like scale with options ranging from "Strongly Disagree" (1 point), "Disagree" (2 points), "Agree" (3 points) to "Strongly Agree" (4 points). The total score ranged from 10 to 40 . Five item scores were reversed so that higher scores of all items in the scale indicated lower self-esteem. The scale had good psychometric properties [34] and was validated among 300 male and female senior secondary adolescents randomly selected from 15 secondary schools in Ile-Ife, Nigeria. 
The test-retest reliability score ranged from 0.85 to 0.88 [35], but no Cronbach's alpha score had been previously reported among Nigerian children and adolescents.

Resilience was assessed with the 10-item ConnorDavidson resilience scale with each item rated on a 5 -point scale from 0 ('not true at all') to 4 ('true nearly all the time'). Possible total scores range from 0 to 40 with higher scores indicating higher resilience [36]. The scale had good psychometric properties for use in various cultures $[37,38]$. It was validated with nursing students in Nigeria, with a Cronbach's alpha of 0.81 [39], but it has not been previously used among Nigerian children and adolescents.

Social support was assessed with the 12-item multidimensional perceived social support scale [40]. The scale has three subscales that assess an individual's perception of the adequacy of their support from family, friends, and significant others. Each subscale comprises four questions. Each item is rated on a 7-point Likert-like scale ranging from 1-"very strongly agree" to 7-"very strongly disagree." The possible total scores range from 12 to 84 , with higher total scores corresponding to higher levels of perceived social support and lower scores corresponding to perceived unavailability or lack of social support. The scale had a Cronbach's alpha of 0.80 when administered to secondary school students in semi-urban Southeast Nigeria [17]; a Cronbach's alpha of 0.78 when administered to stroke survivors attending rehabilitation services in Kano, Northern Nigeria [41]; and a Cronbach's alpha of 0.67 when administered to Orthopaedic patients in Enugu, Southeast Nigeria [42].

\section{Data analysis}

The internal consistency was determined using Cronbach's alpha for the 10-item ACE Questionnaire, victimization subscale of the Illinois Bully Scale, 10-item Rosenberg's self-esteem scale, 10-item Connor-Davidson resilience scale, and the 12-item multidimensional perceived social support scale. Pearson's correlation coefficient $(r)$ was calculated to determine correlations among the scales. Statistical analyses were conducted with Stata/
SE 14.0 for Windows (2015) and significance was set at $p<0.05$.

\section{Results}

The study population consisted of 1001 pupils, of which $549(54.8 \%)$ were girls and $962(96.1 \%)$ were $11-16$ years old. The mean (standard deviation-SD) age was 13.4 (1.77) years. Cronbach's alphas were 0.67 (95\% CI 0.64, 0.70) for the ACE Questionnaire; 0.79 (95\% CI 0.77, 0.81) for the victimization subscale of the Illinois Bully Scale; 0.60 (95\% CI 0.56, 0.64) for the 10-item Rosenberg's selfesteem scale; $0.81(95 \%$ CI $0.79,0.83)$ for the 10 -item Connor-Davidson resilience scale; and 0.93 (95\% CI 0.92, 0.94 ) for the 12-item multi-dimensional perceived social support scale.

Table 1 shows the correlation between the psychosocial factors. All the factors had low but statistically significant correlations with one another. The directions of these correlations differed, however: The direction of the correlation between social support and ACE and bully victimization were inverse; the higher the perceived social support, the lower the number of ACE $(r=-0.21)$, and the lower the bully victimization $(r=-0.16)$. In contrast, the higher the social support, the higher the self-esteem $(r=-0.29)$ and the higher the resilience $(r=0.15)$.

Higher resilience was significantly associated with fewer ACE $(r=-0.07)$, higher self-esteem $(r=-0.21)$ and higher bully victimization $(r=0.13)$. Self-esteem were significantly and directly correlated with the number of ACE and with bully victimization: self-esteem was lower among participants with more ACE $(r=0.25)$ and those with higher bully victimization $(r=0.16)$. Similarly, bully victimization significantly increased as the number of ACE increased $(r=0.20)$.

Cronbach's alphas for the victimization subscale of the Illinois Bully Scale, the 10-item Connor-Davidson resilience scale, and the 12-item multidimensional perceived social support scale were high and compared well with values previously obtained in Nigeria, despite being administered to a more diverse population than our current study population [39, 41, 42]. This similarity suggests that the internal consistency

Table 1 Correlation between the psychosocial variables

\begin{tabular}{lccc}
\hline Psychosocial factors & ACE & Bully victimization & Self-esteem \\
\hline Bully victimization & $0.20^{* * *}$ & & \\
Self esteem & $0.25^{* *}$ & $0.16^{* * *}$ & $-0.21^{* * *}$ \\
Resilience & $-0.07^{*}$ & $0.13^{* * *}$ & $-0.29^{* * *}$ \\
Social support & $-0.21^{* * *}$ & $-0.16^{* * *}$ & $0.15^{* * *}$ \\
\hline
\end{tabular}

${ }^{*} p<0.05,{ }^{* * *} p<0.001$ 
of these instruments is stable across various populations. The Cronbach's alpha values for the ACE questionnaire and the 10-item Rosenberg's self-esteem scale were moderate. These estimates from the present study are the first Cronbach's alpha values for these instruments to be reported among children and adolescents in Nigeria.

There were significant relationships between the study variables as predicted by our hypothesis, though bully victimization correlated positively with resilience. Though it has been suggested that low levels of stress can trigger adaptive coping strategies, which may increase resilience [43], the study finding of a positive correlation between bully victimization and resilience needs to be specifically tested.

In contrast, the negative association between resilience and ACE suggests different mechanisms of protection conferred by resilience for different childhood experiences. Considering the positive associations between resilience, self-esteem and social support found in the present study, it is also possible that resilience and social support together minimize the adverse impacts of stresses in children and adolescents. However, these protective effects may be stronger for bully victimization while the adverse impacts of ACE on children and adolescents' resilience may be relatively more severe. For example, children may feel less threatened by bullying from their peers compared to maltreatment from older adults, over which they may have less control, but this possibility needs to be explored. The association between low social support and increased bully victimization suggests that the isolation associated with lower social support [44] may make affected children targets for bully victimization. It is important, however, to interpret these findings cautiously as this was a cross-sectional study.

Self-esteem score directly correlated with ACE and bully victimization indicating a negative relationship in view of the reverse scoring system for self-esteem: children exposed to more ACE and bully victimization had lower self-esteem than did others not exposed or exposed less to ACE and bully victimization. This finding corroborates past observations [45] and raises concern about the mental health problems of those exposed to ACE and bully victimization. Possible mental health problems resulting from low self-esteem include problems with friendships and romantic relationships, impaired academic and job performance, and increased vulnerability to drug and alcohol abuse [46]. Harnessing the potential of the social support systems in these communities to ameliorate the impact of ACE and bully victimization is as important as instituting efforts to reduce children's experiences of these events.

\section{Limitations}

The ACE Questionnaire and the 10-item Rosenberg's self-esteem scale had moderate internal consistency, so they may require modification or cultural adaptation to improve their Cronbach's alpha scores. In addition, though the Cronbach's alpha provides an estimate of internal consistency, it does not provide an exhaustive indication of a measure's psychometric properties [47]. However, considering that the assessed constructs are relevant for health research and policy development, our findings offer preliminary evidence of acceptable reliability, which can be investigated in future studies. Furthermore, the instruments need to be translated into the main languages used in Nigeria to make them more useable among non-literate Nigerians.

\section{Abbreviation}

ACE: Adverse childhood experiences.

\section{Acknowledgements}

We acknowledge the contributions of the study participants who took the time to respond to questions.

\section{Authors' contributions}

MOF designed the study. OA collected the data. MOF, OO designed the data analysis plan. MOF developed the first draft of the manuscript. MOF, OA, OO and MET contributed to the development of the full manuscript and agreed to the final version of the manuscript and its submission for publication. All authors read and approved the final manuscript.

\section{Funding}

Not applicable.

Availability of data and materials

The data for the study have all been reported in the manuscript.

\section{Ethics approval and consent to participate}

Ethics approval for the study was obtained from the Research and Ethics Committee of the Obafemi Awolowo University Teaching Hospitals Complex, Ile-Ife, Nigeria (ERC/2018/08/06). The study was conducted in line with the requirements of the Declaration of Helsinki. The primary study received written consent from the parents of all study participants. Written assent was obtained from children ages 12-16 years in addition to the parental consent.

\section{Consent to publish}

Not applicable.

\section{Competing interests}

MOF is a Sectional Editor with the BMC Oral Health. MET is an Associate Editor with the BMC Oral Health. All other authors declare that they have no competing interests.

\section{Author details \\ ${ }_{1}^{1}$ Obafemi Awolowo University Teaching Hospitals Complex, Ile-Ife, Nigeria ${ }^{2}$ Department of Child Dental Health, Obafemi Awolowo University, Ile-Ife, Nigeria. ${ }^{3}$ Faculty of Dentistry, Alexandria University, Alexandria, Egypt.}

Received: 24 April 2020 Accepted: 4 July 2020

Published online: 10 July 2020 


\section{References}

1. Baranne ML, Falissard B. Global burden of mental disorders among children aged 5-14 years. Child Adolesc Psychiatry Ment Health. 2018;12(12):19.

2. Kessler RC, McLaughlin KA, Green JG, Gruber MJ, Sampson NA, Zaslavsky AM, et al. Childhood adversities and adult psychopathology in the WHO World Mental Health Surveys. Br J Psychiatry. 2010;197:378-85.

3. McLaughlin KA, Hatzenbuehler ML. Mechanisms linking stressful life events and mental health problems in a prospective, community-based sample of adolescents. J Adolesc Health. 2009;44:153-60.

4. McLaughlin KA, Hatzenbuehler ML, Hilt LM. Emotion dysregulation as a mechanism linking peer victimization to the development of internalizing symptoms among youth. J Consult Clin Psychol. 2009:77:894-904.

5. McLaughlin KA, Green JG, Gruber MJ, Sampson NA, Zaslavsky A, Kessler RC. Childhood adversities and first onset of psychiatric disorders in a national sample of adolescents. Arch Gen Psychiatry. 2012;69:1151-60.

6. McLaughlin KA, Koenen KC, Hill E, Petukhova M, Sampson NA, Zaslavsky A, Kessler RC. Trauma exposure and posttraumatic stress disorder in a US national sample of adolescents. J Am Acad Child Adolesc Psychiatry. 2013:52:815-30.

7. Carliner H, Keyes KM, McLaughlin KA, Meyers JL, Dunn EC, Martins SS. Childhood trauma and illicit drug use in adolescence: a population-based National Comorbidity Survey Replication-Adolescent Supplement study. J Am Acad Child Adolesc Psychiatry. 2016:55:701-8.

8. Klomek AB, Sourander A, Niemelä S, Kumpulainen K, Piha J, Tamminen T, et al. Childhood bullying behaviors as a risk for suicide attempts and completed suicides: a population-based birth cohort study. J Am Acad Child Adolesc Psychiatry. 2009;48:254-61.

9. Copeland WE, Wolke D, Angold A, Costello EJ. Adult psychiatric outcomes of bullying and being bullied by peers in childhood and adolescence. JAMA Psychiatry. 2013;70:419-26.

10. Sourander A, Rönning J, Brunstein-Klomek A, Gyllenberg D, Kumpulainen K, Niemelä S, et al. Childhood bullying behavior and later psychiatric hospital and psychopharmacologic treatment: findings from the Finnish 1981 birth cohort study. Arch Gen Psychiatry. 2009;66:1005-12.

11. Henriksen IO, Ranøyen I, Indredavik MS, Stenseng F. The role of selfesteem in the development of psychiatric problems: a three-year prospective study in a clinical sample of adolescents. Child Adolesc Psychiatry Ment Health. 2017;11:68.

12. Arseneault $L$. The long-term impact of bullying victimization on mental health. World Psychiatry. 2017;16(1):27-8.

13. Lee JH, Nam SK, Kim AR, et al. Resilience: a meta-analytic approach. J Couns Dev. 2013;91:269-79.

14. Johnston MC, Porteous T, Crilly MA, et al. Physical disease and resilient outcomes: a systematic review of resilience definitions and study methods. Psychosomatics. 2015;56:168-80.

15. Hu T, Zhang D, Wang J. A meta-analysis of the trait resilience and mental health. Pers Individ Dif. 2015;76:18-27.

16. Brummett BH, Mark DB, Siegler IC, Williams RB, Babyak MA, Clapp-Channing NE, Barefoot JC. Perceived social support as a predictor of mortality in coronary patients: effects of smoking, sedentary behavior, and depressive symptoms. Psychosom Med. 2005;67(1):40-5.

17. Eze JE, Chukwuorji JC, Ettu PC, Zacchaeus EA, lorfa SK, Nwonyi SK. Bullying and suicide ideation: testing the buffering hypothesis of social support in a sub-Saharan African sample. Journal of Child and Adolescent Trauma. 2019. https://doi.org/10.1007/s40653-019-00294-w (Online ahead of print).

18. McLaughlin K. The long shadow of adverse childhood experiences. Psychological Science Agenda |April 2017. https://www.apa.org/science/ about/psa/2017/04/adverse-childhood. Accessed 14 Apr 2020.

19. Department of Health and Human Services, US Public Health Service. Chapter 2 Culture Counts: The Influence of Culture and Society on Mental Health. In: Mental Health: Culture, Race, and Ethnicity: a Supplement to Mental Health: A Report of the Surgeon General. 2001

20. Yetim U. The impacts of individualism/collectivism, self-esteem, and feeling of Mastery on Life Satisfaction among the Turkish University students and academicians. Soc Indic Res. 2003;61:297-317.

21. Oladeji BD, Makanjuola VA, Gureje O. Family-related adverse childhood experiences as risk factors for psychiatric disorders in Nigeria. Br J Psychiatry. 2010;196(3):186-91.
22. Fenny O, Falola MI. Prevalence and correlates of bullying behavior among Nigerian middle school students. Int J Offender Ther Comp Criminol. 2020;64(5):564-85.

23. Akanni OO, Olashore AA, Osasona SO, Uwadiae E. Predictors of bullying reported by perpetrators in a sample of senior school students in Benin City, Nigeria. S Afr J Psychiatr. 2020;26:1359.

24. Chinawa JM, Obu HA, Manyike PC, Obi IE, Isreal OO, Chinawa AT. Self esteem among adolescents in Nigerian secondary schools: a neglected issue. J Adv Med Med Res. 2014:5(1):98-106.

25. Aluh DO, Anyachebelu OC, Anosike C, Anizoba EL. Mental health literacy: what do Nigerian adolescents know about depression? Int J Ment Health Syst. 2018;12:8. https://doi.org/10.1186/s13033-018-0186-2.

26. Chambers $C T$, Johnston C. Developmental differences in children's use of rating scales. J Pediatr Psychol. 2002;27(1):27-36.

27. Harter S, Whitesell NR. Developmental changes in children's understanding of single, multiple, and blended emotion concepts. In: Saarni C, Harris $\mathrm{PL}$, editors. Children's understanding of emotion. Cambridge: Cambridge University Press; 1989. p. 81-116.

28. Crouch E, Probst JC, Radcliff E, Bennett KJ, McKinney SH. Prevalence of adverse childhood experiences (ACEs) among US children. Child Abuse Negl. 2019;92:209-18.

29. Metcalfe C. Biostatistics: A foundation for analysis in the health sciences. 7th edition. Wayne W. Daniel, Wiley. Stat Med. 2001; 20(2):324-326.

30. Adekoya-Sofowora C, Nasir W, Oginni A, et al. Dental caries in 12-year-old suburban Nigerian school children. Afr Health Sci. 2006;6(3):145-50.

31. Felitti VJ, Anda RF, Nordenberg D, Williamson DF, Spitz AM, Edwards V, et al. Relationship of childhood abuse and household dysfunction to many of the leading causes of death in adults. The Adverse Childhood Experiences (ACE) Study. Am J Prev Med. 1998;14(4):245-58.

32. Wingenfeld K, Schäfer I, Terfehr K, Grabski H, Driessen M, Grabe H, et al. The reliable, valid and economic assessment of early traumatization: first psychometric characteristics of the German version of the Adverse Childhood Experiences Questionnaire (ACE). Psychother Psychosom Med Psychol. 2011;61(1):e10-4.

33. Espelage DL, Holt M. Bullying and victimization during early adolescence: peer influences and psychosocial correlates. J Emot Abus. 2001;2:123-42.

34. Rosenberg M. Society and the adolescent self-image. Princeton: Princeton University Press; 1965

35. Okoiye OE, Anyaochi NN, Onah AT. Moderating effect of cyberbullying on the psychological well-being of in-school adolescents in Benin Edo State Nigeria. Eur J Sustain Dev. 2015;4(1):109-18.

36. Campbell-Sills L, Stein MB. Psychometric analysis and refinement of the Connor-Davidson Resilience Scale (CD-RISC): validation of a 10-item measure of resilience. J Trauma Stress. 2007;20(6):1019-28.

37. Notario-Pacheco B, Solera-Martínez M, Serrano-Parra MD, BartoloméGutiérrez R, García-Campayo J, Martínez-Vizcaíno V. Reliability and validity of the Spanish version of the 10-item Connor-Davidson Resilience Scale (10-item CD-RISC) in young adults. Health Qual Life Outcomes. 2011;9:63.

38. Wang L, Shi Z, Zhang Y, Zhang Z. Psychometric properties of the 10-item Connor-Davidson Resilience Scale in Chinese earthquake victims. Psychiatry Clin Neurosci. 2010:64(5):499-504.

39. Aloba O, Olabisi O, Aloba T. The 10-Item Connor-Davidson resilience scale: factorial structure, reliability, validity, and correlates among student nurses in Southwestern Nigeria. J Am Psychiatr Nurses Assoc. 2016:22(1):43-51.

40. Zimet GD, Dahlem NW, Zimet SG, Gordon K. The multidimensional scale of perceived social support. J Pers Assess. 1988;52:30-41.

41. Mohammad AH, Al Sadat N, Yim LS, Chinna K. Validity and Reliability of the Hausa version of multidimensional scale of perceived social support index. Iran Red Crescent Med J. 2015:17(2):e18776.

42. Ifeagwazi CM, Obi NG, Udensi CE, Chukwuorji JC. Influence of perceived injustice, social support and gender on depressive symptoms among orthopaedic patients. Eur J Sci Res. 2014;125(3):370-83.

43. Rutter M. Psychosocial resilience and protective mechanisms. Amer J Orthopsychiat. 1987;57(3):316-31.

44. Tomaka J, Thompson S, Palacios R. The relation of social isolation, Ioneliness, and social support to disease outcomes among the elderly. J Aging Health. 2006;18(3):359-84. https://doi.org/10.1177/0898264305280993.

45. Khodabandeh F, Khalilzadeh M, Hemati Z. The impact of adverse childhood experiences on adulthood aggression and self-esteem-a study on male forensic clients. Nov Biomed. 2018:2:85-91. 
46. Mann M, Hosman CM, Schaalma HP, de Vries NK. Self-esteem in a broadspectrum approach for mental health promotion. Health Educ Res. 2004;19(4):357-72.

47. Sijtsma K. On the use, the misuse, and the very limited usefulness of Cronbach's alpha. Psychometrika. 2009;74(1):107-20.

\section{Publisher's Note}

Springer Nature remains neutral with regard to jurisdictional claims in published maps and institutional affiliations.
Ready to submit your research? Choose BMC and benefit from:

- fast, convenient online submission

- thorough peer review by experienced researchers in your field

- rapid publication on acceptance

- support for research data, including large and complex data types

- gold Open Access which fosters wider collaboration and increased citations

- maximum visibility for your research: over 100M website views per year

At BMC, research is always in progress.

Learn more biomedcentral.com/submissions 